\title{
Addressing a single spin in diamond with a macroscopic dielectric microwave cavity
}

J.-M. Le Floch, C. Bradac, N. Nand, S. Castelletto, M. E. Tobar, and T. Volz

Citation: Appl. Phys. Lett. 105, 133101 (2014); doi: 10.1063/1.4896858

View online: $\mathrm{http}: / / d x . d o i . o r g / 10.1063 / 1.4896858$

View Table of Contents: http://aip.scitation.org/toc/apl/105/13

Published by the American Institute of Physics

\section{Articles you may be interested in}

Towards achieving strong coupling in three-dimensional-cavity with solid state spin resonance Journal of Applied Physics 119, 153901 (2016); 10.1063/1.4946893

Broadband, large-area microwave antenna for optically detected magnetic resonance of nitrogen-vacancy centers in diamond

Review of Scientific Instruments 87, 053904 (2016); 10.1063/1.4952418

High-frequency and high-field optically detected magnetic resonance of nitrogen-vacancy centers in diamond Applied Physics Letters 106, 063111 (2015); 10.1063/1.4908528

Single spin optically detected magnetic resonance with $60-90 \mathrm{GHz}$ (E-band) microwave resonators Review of Scientific Instruments 86, 064704 (2015); 10.1063/1.4922664

Nanoscale magnetic field mapping with a single spin scanning probe magnetometer Applied Physics Letters 100, 153118 (2012); 10.1063/1.3703128

Inhomogeneous broadening of optically detected magnetic resonance of the ensembles of nitrogen-vacancy centers in diamond by interstitial carbon atoms

Applied Physics Letters 106, 102402 (2015); 10.1063/1.4913428

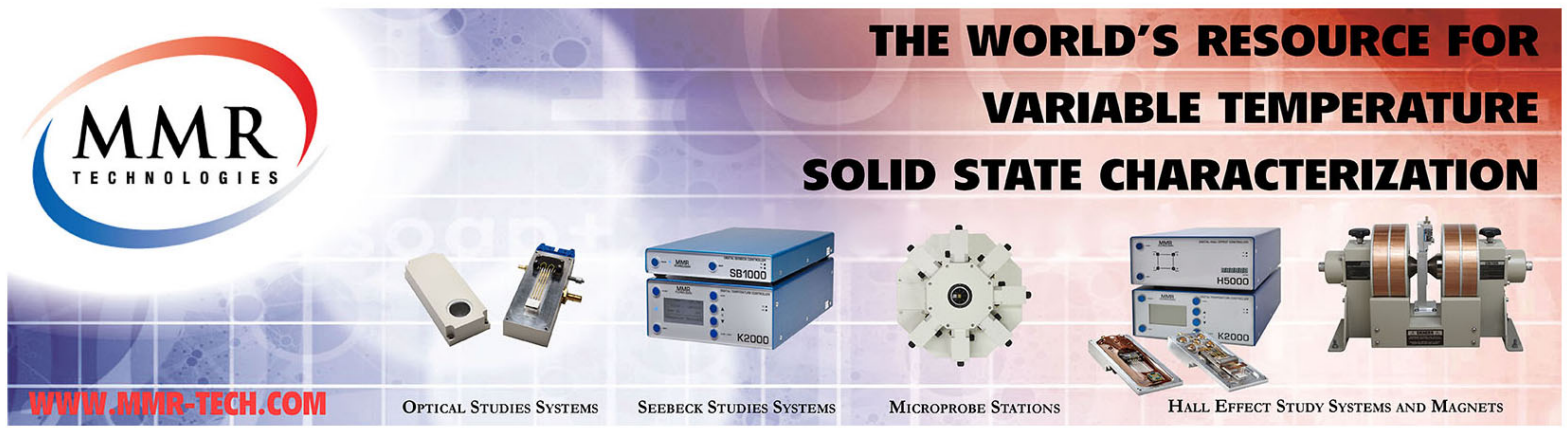




\title{
Addressing a single spin in diamond with a macroscopic dielectric microwave cavity
}

\author{
J.-M. Le Floch, ${ }^{1}$ C. Bradac,${ }^{2}$ N. Nand,${ }^{2}$ S. Castelletto, ${ }^{3}$ M. E. Tobar, ${ }^{1}$ and T. Volz ${ }^{2}$ \\ ${ }^{1}$ ARC Centre of Excellence for Engineered Quantum Systems, School of Physics, The University of Western \\ Australia, Crawley, Western Australia 6009, Australia \\ ${ }^{2}$ ARC Centre of Excellence for Engineered Quantum Systems, Department of Physics and Astronomy, \\ Macquarie University, North Ryde, New South Wales 2109, Australia \\ ${ }^{3}$ School of Aerospace, Mechanical and Manufacturing Engineering, RMIT University, Melbourne, Australia
}

(Received 19 August 2014; accepted 19 September 2014; published online 29 September 2014)

We present a technique for addressing single nitrogen-vacancy (NV) center spins in diamond over macroscopic distances using a tunable dielectric microwave cavity. We demonstrate optically detected magnetic resonance (ODMR) for a single negatively charged $\mathrm{NV}$ center $\left(\mathrm{NV}^{-}\right)$in a nanodiamond (ND) located directly under the macroscopic microwave cavity. By moving the cavity relative to the ND, we record the ODMR signal as a function of position, mapping out the distribution of the cavity magnetic field along one axis. In addition, we argue that our system could be used to determine the orientation of the $\mathrm{NV}^{-}$major axis in a straightforward manner. (C) 2014 AIP Publishing LLC. [http://dx.doi.org/10.1063/1.4896858]

Over the past decade, nitrogen-vacancy (NV) color centers in diamond (Figure 1(a)) have attracted a great deal of interest due to their outstanding quantum properties. ${ }^{1}$ Experiments have demonstrated long ground-state spin coherence times of the negatively charged $\mathrm{NV}$ center $\left(\mathrm{NV}^{-}\right)$even at room temperature. ${ }^{2}$ This makes $\mathrm{NV}^{-}$centers in diamond ideal candidates for room-temperature qubits ${ }^{3,4}$ and for ultrasensitive quantum sensors for detecting magnetic ${ }^{5-11}$ and electric fields ${ }^{12}$ at the nanoscale even in biological settings. ${ }^{9,13,14}$

Both quantum information processing and quantum sensing with $\mathrm{NV}^{-}$spins require the (coherent) manipulation and addressing of individual spins typically through the application of microwave (MW) radiation at a frequency that is resonant with the ground-state spin transition. The $\mathrm{NV}^{-}$exhibits a zero-field spin resonance at $2.87 \mathrm{GHz}$, which occurs between the $m_{s}=0$ and $m_{s}= \pm 1$ spin sublevels of its spin triplet ground state (see Figure 1(b)). The most commonly used approaches for applying microwaves at this frequency are onchip microstrip lines (thin wires), coplanar waveguides, or free-space loop antennas. On-chip solutions rely on near-field coupling and require the $\mathrm{NV}^{-}$spin to be in close proximity (on the order of $10 \mu \mathrm{m}$ ) to the wire or microstrip line. Besides the inhomogeneity of the field, these on-chip solutions can easily lead to significant sample heating and undesired sample drift. Loop antennas typically work in the far field but require orders of magnitude larger amount of radiated MW power.

In order to address single $\mathrm{NV}^{-}$spins in diamond, we designed a so-called dielectric-loaded microwave resonator (DLR) with high quality (Q) factor (see Figure 1(c)). DLRs of this kind are typically used in low-temperature electron paramagnetic resonance experiments for measuring the complex permittivity of extremely low-loss dielectrics, ${ }^{15-17}$ but are also employed for testing local Lorentz invariance in fundamental experiments. ${ }^{18}$ In industrial settings, DLRs find applications in radar, proximity detection, information transmission, remote guiding, navigation, and positioning. ${ }^{19,20}$ In order to find an appropriate design for our DLR, we employed the numerical method of lines ${ }^{21}$ or finite element analysis. The design was guided by the desire to have compact cavity dimensions and the requirement for the field to couple evanescently to the $\mathrm{NV}^{-}$spins located in close vicinity under the cavity.

We found the best configuration to be an open cylindrically symmetric cavity with a pure transverse electric (TE) mode with two non-vanishing magnetic-field components, $\mathrm{H}_{r}$ and $\mathrm{H}_{z}$. In contrast to whispering gallery modes, the TEfield confinement into the dielectric is not as high and exhibits less spurious modes, leading to a higher Q-factor. ${ }^{22} \mathrm{We}$ denote the different cavity modes by $\mathrm{TE}_{m, n, p}$, where $2 m$ is the number of azimuthal nodes, $n$ is the number of radial nodes, and $p$ is the number of nodes along the z-axis (symmetry axis) of the cylinder. For pure TE-modes, the

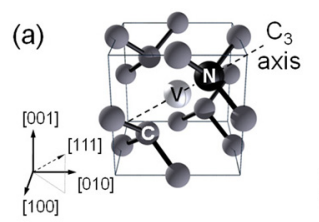

(d) -25

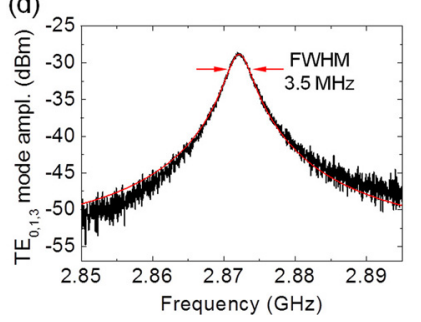

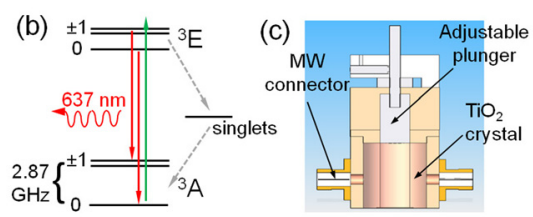

(e)

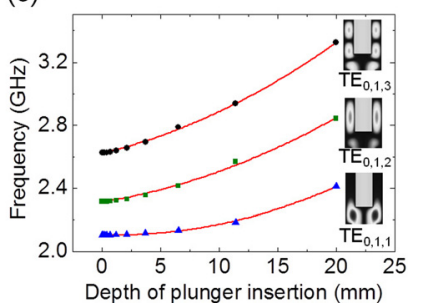

FIG. 1. (a) Diamond lattice structure with an embedded NV center. (b) Level scheme for the $\mathrm{NV}^{-}$center including the hyperfine splitting of the triplet ground state $\left({ }^{3} \mathrm{~A}\right)$. Optical pumping of the spin into the $m_{S}=0$ state occurs via an intersystem crossing to the singlet manifold. (c) Dielectric cavity with adjustable plunger. The outer diameter of the cavity is $32 \mathrm{~mm}$, its height amounts to $20 \mathrm{~mm}$. (d) Transmission spectrum of the cavity with a Full-Width Half-Maximum (FWHM) of $3.5 \mathrm{MHz}$ corresponding to a Q-factor of around 1000. The spectrum was recorded using a Fieldfox N9918A (Agilent Technologies). (e) Numerically calculated cavity frequency as a function of plunger position. The mode of interest $\mathrm{TE}_{0,1,3}$ tunes easily across the $\mathrm{NV}^{-}$ground-state spin transition. 
azimuthal mode number vanishes, i.e., $m=0$. Due to the particular boundary conditions, they only have three nonvanishing components of the electromagnetic field, $E_{\Theta}, H_{r}$, and $H_{z}$. By inserting a dielectric rod made of highpermittivity, low-loss microwave material, the field can be confined to an area of roughly $10 \times 10 \mathrm{~mm}^{2}$. From the few suitable materials available, ${ }^{23}$ we chose $\mathrm{TiO}_{2}$ for which the fundamental mode has a frequency of $2.2 \mathrm{GHz}$. For addressing the $\mathrm{NV}^{-}$spin transition, we then use the higher-order $\mathrm{TE}_{0,1,3}$-mode resonating at $2.7 \mathrm{GHz}$. Frequency tuning is achieved by mechanical insertion of a metallic plunger, which directly affects the electric field and shifts the resonance frequency up to a value of $3.1 \mathrm{GHz}$ (Figure 1(e)). The (loaded) Q-factor of this cavity mode is about 1000 (see Figure 1(d)). Note that for perfect input coupling we would expect the circulating intra-cavity power to be enhanced by a factor Q compared to the incoming MW power. However, in the current cavity the coupling coefficient is rather small (about $1 \%$ ).

The magnetic field of the DLR cavity has cylindrical symmetry. Both the radial magnetic field strength, $H_{r}$, and the vertical magnetic field strength, $H_{z}$, are displayed in Figure 2. Figures 2(a) and 2(b) show two-dimensional plots of the respective field strength in a plane that contains the symmetry axis of the cavity: The $\mathrm{x}$-axis corresponds to the radial distance from the symmetry axis and the values on the $y$-axis indicate the height above the bottom edge of the cavity. We note that the radial field component $H_{r}$ has a maximum right at the bottom edge at $r \approx 7 \mathrm{~mm}$, indicating a strong evanescent component. The vertical field $H_{z}$ is well-contained within the cavity. Figures $2(\mathrm{c})$ and $2(\mathrm{~d})$ show the expected variation of the radial and vertical field in a plane $1 \mathrm{~mm}$ below the cavity. The plots are normalized to the local overall field strength $\sqrt{H_{r}^{2}+H_{z}^{2}}$. The graphs clearly show that in the center of the cavity the $H_{z}$ component dominates (due to symmetry) whereas right under the (a)

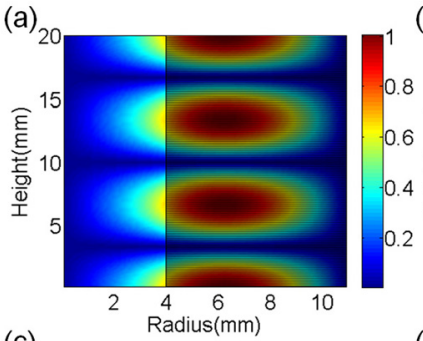

(c)

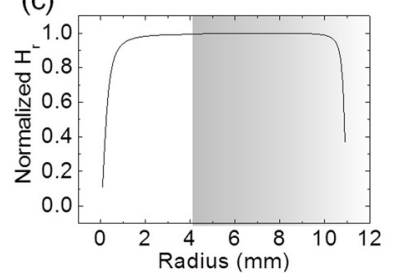

(b)

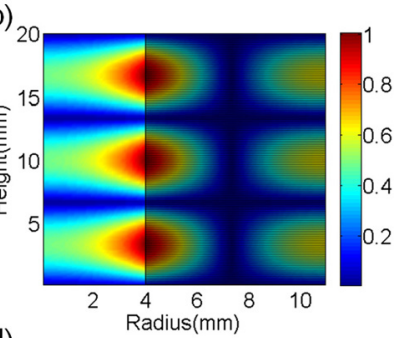

(d)

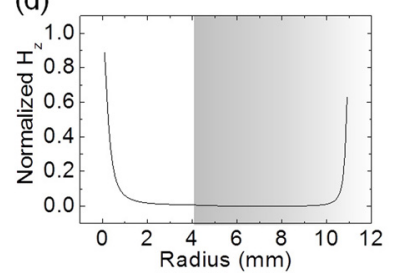

FIG. 2. (a) Radial magnetic field strength $H_{r}$ in a two-dimensional cut along the vertical symmetry axis (y-axis of the graph) of the microwave cavity. (b) Analogous plot for the strength of the vertical magnetic field $H_{z}$. Shaded areas indicate the dielectric material of the cavity. (c) and (d) Field intensities $H_{r}$ and $H_{z}$ along the radial direction in a plane $1 \mathrm{~mm}$ below the cavity. Both fields are normalized to the local overall field strength $\sqrt{H_{r}^{2}+H_{z}^{2}}$. In the center of the trap, only $H_{z}$ is non-vanishing whereas directly under the dielectric (shaded region) $H_{r}$ is the dominant field component. dielectric at $r \approx 7 \mathrm{~mm}$, the radial field is the dominant component.

We now move on to demonstrate optically detected magnetic resonance (ODMR) of a single $\mathrm{NV}^{-}$spin located just below the cavity. The HPHT nanodiamonds (NDs) (MSY $0.1 \mu \mathrm{m}$, Microdiamant) are placed on a glass coverslip approximately $1 \mathrm{~mm}$ below the cavity which is mounted on a $x-y-z$ mechanical stage (see Figure 3(a)). The ND fluorescence upon excitation with a $532 \mathrm{~nm}$ laser is collected using a home-built confocal microscope ${ }^{24}$ and sent to either a spectrometer or to avalanche photodetectors. Once a suitable single $\mathrm{NV}^{-}$center is identified, we obtain an ODMR signal by applying microwave radiation through our microwave cavity and recording the corresponding fluorescence as a function of microwave frequency. The microwave signal is generated using a standard microwave generator (SMIQ 06B, Rohde \& Schwarz) and amplified (25S1G4A, Amplifier Research) before applying it to the cavity. A typical ODMR signal is displayed in Figure 3(b), clearly demonstrating the coupling of a single $\mathrm{NV}^{-}$spin to the macroscopic microwave resonator. Note that the contrast of the ODMR signal was optimized by adjusting the cavity resonance frequency to the actual transition frequency of the selected $\mathrm{NV}^{-}$center. Depending on the ND, we found a maximum achievable contrast of up to $12 \%$. Next, we recorded a saturation curve for the $m_{s}=0 \rightarrow m_{s}= \pm 1$ transition 3(c) giving a saturation power of about $5 \mathrm{dBm}$ for this particular $\mathrm{NV}^{-}$spin.

In order to demonstrate the spatial variation of the magnetic field, we recorded an ODMR signal as a function of relative position between the ND and the center axis of the cavity by mechanically adjusting the cavity position. In the low-power (or linear) regime, the contrast of the ODMR signal measures the local microwave power seen by the $\mathrm{NV}^{-}$

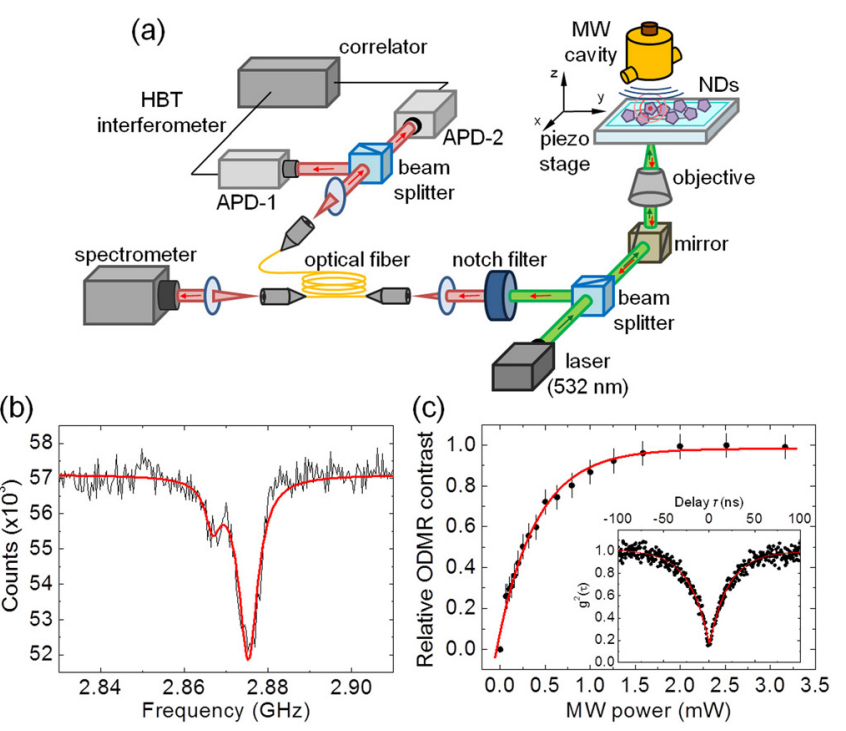

FIG. 3. (a) Experimental setup for measuring ODMR with the dielectric MW cavity. The ND fluorescence is collected from below using a confocal setup. The collected photons are sent to either a spectrometer or to avalanche photodetectors. (b) Single-spin ODMR signal for a ND in the dielectric MW cavity. (c) Saturation curve for the ODMR contrast as a function of microwave power (produced by the signal generator). The inset shows an autocorrelation signal with clear antibunching demonstrating that the ND contains a single $\mathrm{NV}^{-}$center. 


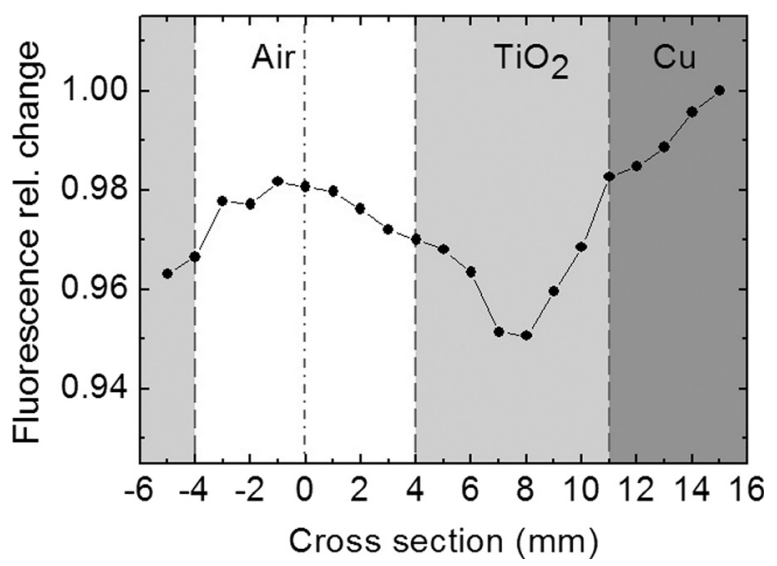

FIG. 4. ODMR contrast as a function of ND position relative to the cavity axis. The maximum contrast is observed directly under the dielectric material in agreement with the expected field dependence from Figure 2. At the center of the cavity $(r=0)$, the contrast does not completely vanish due to the finite value of $H_{z}$.

spin. Figure 4 shows the result of such a measurement taken along the $\mathrm{x}$-axis in consecutive 1-mm steps, while the $\mathrm{z}$ - and $y$-coordinate of the resonator were kept fixed with respect to the ND position. In the figure, we plot the depth of the ODMR resonance as a function of position, normalized to 1 . The plot displays the expected variation in ODMR contrast and exhibits a maximum contrast of about $6 \%$ when the ND is right below the dielectric of the cavity structure at $r \approx$ $7 \mathrm{~mm}$. The finite contrast at $r=0$ indicates that the $\mathrm{NV}^{-}$spin has a non-vanishing in-plane component. Since we do not know the major axis of the $\mathrm{NV}^{-}$center, we cannot extract the full information about the cavity magnetic field from Figure 4. Using a ND with a known spin orientation, however, magnetic-field tomography is possible. The cavity presented here could also be used as a tool to determine the major axis of a single $\mathrm{NV}^{-}$center in a straightforward manner: By measuring the ODMR contrast in the center of the cavity and in two additional points along the circumference just below the dielectric slab at $r \approx 7 \mathrm{~mm}$, the direction of the $\mathrm{NV}^{-}$spin can be calculated-provided the ratio $H_{z}(r=0) /$ $H_{r}(r=7 \mathrm{~mm})$ is known.

The main advantages of our technique are the large area over which the spins can be addressed and the absence of undesired sample heating allowing for stable long-term observations. Our measurements once again demonstrate the potential of $\mathrm{NV}^{-}$centers as robust technologically viable magnetic-field sensors. In addition, the DLR cavity can serve as a tool for identifying the orientation of $\mathrm{NV}^{-}$spins. In the future, we plan to use the device for coherent time-resolved spin manipulation.

This work was supported by the Australian Research Council Centre of Excellence CE110001013 and a
University of Western Australia (UWA) research collaboration award.

${ }^{1}$ M. W. Doherty, N. B. Manson, P. Delaney, F. Jelezko, J. Wrachtrup, and L. C. L. Hollenberg, Phys. Rep. 528, 1 (2013).

${ }^{2}$ G. Balasubramanian, P. Neumann, D. Twitchen, M. Markham, R. Kolesov, N. Mizuochi, J. Isoya, J. Achard, J. Beck, J. Tissler, V. Jacques, P. R. Hemmer, F. Jelezko, and J. Wrachtrup, Nat. Mater. 8, 383-387 (2009).

${ }^{3}$ M. V. Gurudev Dutt, L. Childress, L. Jiang, E. Togan, J. Maze, F. Jelezko, A. S. Zibrov, P. R. Hemmer, and M. D. Lukin, Science 316, 1312-1316 (2007).

${ }^{4}$ P. Neumann, N. Mizuochi, F. Rempp, P. Hemmer, H. Watanabe, S. Yamasaki, V. Jacques, T. Gaebel, F. Jelezko, and J. Wrachtrup, Science 320, 1326-1329 (2008).

${ }^{5}$ G. Balasubramanian1, I. Y. Chan, R. Kolesov, M. Al-Hmoud, J. Tisler, C. Shin, C. Kim, A. Wojcik, P. R. Hemmer, A. Krueger, T. Hanke, A. Leitenstorfer, R. Bratschitsch, F. Jelezko, and J. Wrachtrup, Nature 455, 648-651 (2008).

${ }^{6}$ J. R. Maze, P. L. Stanwix, J. S. Hodges, S. Hong, J. M. Taylor, P. Cappellaro, L. Jiang, M. V. Gurudev Dutt, E. Togan, A. S. Zibrov, A. Yacoby, R. L. Walsworth, and M. D. Lukin, Nature 455, 644-647 (2008).

${ }^{7}$ C. L. Degen, Appl. Phys. Lett. 92, 243111 (2008).

${ }^{8}$ J. M. Taylor, P. Cappellaro, L. Childress, L. Jiang, D. Budker, P. R. Hemmer, A. Yacoby, R. Walsworth, and M. D. Lukin, Nat. Phys. 4, 810-816 (2008).

${ }^{9}$ L. P. McGuinness, Y. Yan, A. Stacey, D. A. Simpson, L. T. Hall, D. Maclaurin, S. Prawer, P. Mulvaney, J. Wrachtrup, F. Caruso, R. E. Scholten, and L. C. L. Hollenberg, Nat. Nanotechnol. 6, 358-363 (2011).

${ }^{10}$ M. S. Grinolds, M. Warner, K. De Greve, Y. Dovzhenko, L. Thiel, R. L. Walsworth, S. Hong, P. Maletinsky, and A. Yacoby, Nat. Nanotechnol. 9, 279-284 (2014).

${ }^{11}$ J.-P. Tetienne, T. Hingant, J.-V. Kim, L. Herrera Diez, J.-P. Adam, K. Garcia, J.-F. Roch, S. Rohart, A. Thiaville, D. Ravelosona, and V. Jacques, Science 344, 1366-1369 (2014).

${ }^{12}$ F. Dolde, H. Fedder, M. W. Doherty, T. Nöbauer, F. Rempp, G. Balasubramanian, T. Wolf, F. Reinhard, L. C. L. Hollenberg, F. Jelezko, and J. Wrachtrup, Nat. Phys. 7, 459-463 (2011).

${ }^{13}$ O. Faklaris, D. Garrot, V. Joshi, F. Druon, J. P. Boudou, T. Sauvage, P. Georges, P. A. Curmi, and F. Treussart, Small 4, 2236 (2008).

${ }^{14}$ A. Ermakova, G. Pramanik, J.-M. Cai, G. Algara-Siller, U. Kaiser, T. Weil, Y.-K. Tzeng, H. C. Chang, L. P. McGuinness, M. B. Plenio, B. Naydenov, and F. Jelezko, Nano Lett. 13, 3305-3309 (2013).

${ }^{15}$ M. E. Tobar, J. Krupka, E. N. Ivanov, and R. A. Woode, J. Appl. Phys. 83, 1604 (1998).

${ }^{16}$ J. Krupka, K. Derzakowski, M. E. Tobar, J. Hartnett, and R. G. Geyerk, Meas. Sci. Technol. 10, 387-392 (1999).

${ }^{17}$ J.-M. le Floch, J. D. Anstie, M. E. Tobar, J. G. Hartnett, P.-Y. Bourgeois, and D. Cros, Phys. Lett. A 359, 1-7 (2006).

${ }^{18}$ M. E. Tobar, E. N. Ivanov, P. L. Stanwix, J.-M. Le Floch, and J. G. Hartnett, Phys. Rev. D 80, 125024 (2009).

${ }^{19}$ C. Rieck, P. Jarlemark, R. Emardson, and K. Jaldehag, in Proceedings of the 22nd EFTF, Toulouse, France, 2008.

${ }^{20}$ F. Droz, P. Mosset, G. Barmaverain, P. Rochat, Q. Wang, M. Belloni, L. Mattioni, F. Emma, and P. Waller, in Proceedings of the 20th EFTF, Braunschweig, Germany, 2006.

${ }^{21}$ J.-M. Le Floch, M. E. Tobar, D. Cros, and J. Krupka, IEEE Trans. Ultrason. Ferroelectr. Freq. Control 54, 2689-2695 (2007).

${ }^{22}$ J.-M. Le Floch, Y. Fan, G. Humbert, Q. Shan, D. Férachou, R. BaraMaillet, M. Aubourg, J. G. Hartnett, V. Madrangeas, D. Cros, J.-M. Blondy, J. Krupka, and M. E. Tobar, Rev. Sci. Instrum. 85, 031301 (2014).

${ }^{23}$ J.-M. Le Floch, M. E. Tobar, D. Cros, and J. Krupka, Appl. Phys. Lett. 92, 032901 (2008).

${ }^{24}$ C. Bradac, T. Gaebel, N. Naidoo, M. J. Sellars, J. Twamley, L. J. Brown, A. S. Barnard, T. Plakhotnik, A. V. Zvyagin, and J. R. Rabeau, Nat. Nanotechnol. 5, 345-349 (2010). 